\title{
A PROPERTY OF ANALYTIC FUNCTIONS WITH HADAMARD GAPS
}

\author{
JiE MiAO
}

In this paper we obtain a sufficient and necessary condition for an analytic function $f$ on $D$ with Hadamard gaps, that is, for $f(z)=\sum_{k=1}^{\infty} a_{k} z^{n_{k}}$ satisfying $n_{k+1} / n_{k} \geqslant$ $\lambda>1$ for all $k$, to belong to a kind of space consisting of analytic functions on $D$. The special cases of these spaces are $B M O A$ and $V M O A$. In view of our result we can answer the open question given recently by Stroethoff.

\section{INTRODUCTION}

Let $D=\{z:|z|<1\}$ be the open disc in the complex plane. For an analytic function $f$ on $D$ we set

$$
\|f\|_{B M O A}=\sup _{\lambda \in D}\left(\frac{1}{2 \pi} \int_{0}^{2 \pi}\left|f\left(\varphi_{\lambda}\left(e^{i \theta}\right)\right)-f(\lambda)\right|^{2} d \theta\right)^{1 / 2},
$$

where

$$
\varphi_{\lambda}(z)=\frac{z-\lambda}{1-\bar{\lambda} z}, \quad z \in D .
$$

The space $B M O A$ is the set of all analytic functions $f$ on $D$ for which $\|f\|_{B M O A}<\infty$. Contained in $B M O A$ is the subspace $V M O A$, the set of all analytic functions $f$ on $D$ for which

$$
\lim _{|\lambda| \rightarrow 1-0}\left(\frac{1}{2 \pi} \int_{0}^{2 \pi}\left|f\left(\varphi_{\lambda}\left(e^{i \theta}\right)\right)-f(\lambda)\right|^{2} d \theta\right)=0 .
$$

It is well-known that for every analytic function $f$ on $D$ (see [2]),

$$
\|f\|_{B M O A} \approx \sup _{\lambda \in D}\left(\int_{D}\left|f^{\prime}(z)\right|^{2}\left(1-\left|\varphi_{\lambda}(z)\right|^{2}\right) d A(z)\right)^{\frac{1}{2}}
$$

and $f \in V M O A$ if and only if

$$
\lim _{|\lambda| \rightarrow 1-0} \int_{D}\left|f^{\prime}(z)\right|^{2}\left(1-\left|\varphi_{\lambda}(z)\right|^{2}\right) d A(z)=0
$$

\section{Received 5 February 1991}

This research was partially supported by a grant from NSF of Peoples Republic of China.

Copyright Clearance Centre, Inc. Serial-fee code: 0004-9729/92 \$A2.00+0.00. 
where $A$ denotes the Lebesgue area measure and " $\approx$ " means equivalently (see [4]). The Bloch space $\mathcal{B}$ is the set of all analytic functions $f$ on $D$ for which $\|f\|_{B}=$ $\sup _{z \in D}\left(1-|z|^{2}\right)\left|f^{\prime}(z)\right|<\infty$. Contained in $\mathcal{B}$ is the little Bloch space $\mathcal{B}_{0}$, the set of all analytic functions $f$ on $D$ for which $\lim _{|z| \rightarrow 1-0}\left(1-|z|^{2}\right)\left|f^{\prime}(z)\right|=0$. We know that for $0<p<\infty$ (see [4]),

$$
\|f\|_{B} \approx \sup _{\lambda \in D}\left(\int_{D}\left|f^{\prime}(z)\right|^{p}\left(1-|z|^{2}\right)^{p-2}\left(1-\left|\varphi_{\lambda}(z)\right|^{2}\right)^{2} d A(z)\right)^{1 / p}
$$

and $f \in \mathcal{B}_{0}$ if and only if

$$
\lim _{|\lambda| \rightarrow 1-0} \int_{D}\left|f^{\prime}(z)\right|^{p}\left(1-|z|^{2}\right)^{p-2}\left(1-\left|\varphi_{\lambda}(z)\right|^{2}\right)^{2} d A(z)=0 .
$$

It has been known to us that $\mathcal{B}$ and $B M O A$ share many analogous properties, as do $\mathcal{B}_{0}$ and $V M O A$. Comparing the above equivalence (1) with (3), as well as (2) with (4) when $p=2$, Stroethoff [4] offered the following open question:

QUeSTION: Let $0<p<\infty$ and let $f$ be an analytic function on $D$. Are the following true?

$$
\begin{aligned}
& \text { (i) } f \in B M O A \Longleftrightarrow \sup _{\lambda \in D} \int_{D}\left|f^{\prime}(z)\right|^{p}\left(1-|z|^{2}\right)^{p-2}\left(1-\left|\varphi_{\lambda}(z)\right|^{2}\right) d A(z)<\infty, \\
& \text { (ii) } f \in V M O A \Longleftrightarrow \lim _{\lambda \rightarrow 1-0} \int_{D}\left|f^{\prime}(z)\right|^{p}\left(1-|z|^{2}\right)^{p-2}\left(1-\left|\varphi_{\lambda}(z)\right|^{2}\right) d A(z)=0 .
\end{aligned}
$$

For $p=2$ the above question has a positive answer. Making use of the fact that there is a constant $C$ such that

$$
\|f\|_{B} \leqslant C\|f\|_{B M O A}
$$

for every analytic $f$ on $D$, we know a partial answer to the question: for an analytic function $f$ on $D$ and $0<p \leqslant 2$ the conditions in (i) and (ii) are sufficient for containment in $B M O A$ and $V M O A$, respectively; for $2 \leqslant p<\infty$ the conditions in (i) and (ii) are necessary for $f$ to belong to $B M O A$ and $V M O A$, respectively.

Let $0<p<\infty$. For an analytic function $f$ on $D$ we set

$$
\|f\|_{B^{p}}=\sup _{\lambda \in D}\left(\int_{D}\left|f^{\prime}(z)\right|^{p}\left(1-|z|^{2}\right)^{p-2}\left(1-\left|\varphi_{\lambda}(z)\right|^{2}\right) d A(z)\right)^{1 / p} .
$$


We define the space $B^{p}$ to be the set of all analytic functions $f$ on $D$ for which $\|f\|_{B^{p}}<\infty$ and define $B_{0}^{p}$ to be the subspace of $B^{p}$, the set of all analytic functions $f$ on $D$ for which

$$
\lim _{|\lambda| \rightarrow 1-0} \int_{D}\left|f^{\prime}(z)\right|^{p}\left(1-|z|^{2}\right)^{p-2}\left(1-\left|\varphi_{\lambda}(z)\right|^{2}\right) d A(z)=0 .
$$

It is clear that for $0<p<\infty, B^{p} \subset \mathcal{B}$ and $B_{0}^{p} \subset \mathcal{B}_{0}$, especially $B^{2}=B M O A$, $B_{0}^{2}=V M O A$. The known partial answer now can be expressed as: for $0<p<2$,

$$
B^{p} \subset B M O A, \quad B_{0}^{p} \subset V M O A ;
$$

for $2<p<\infty$,

$$
B M O A \subset B^{q}, \quad V M O A \subset B_{0}^{q} .
$$

According to our definition, Stroethoff's question becomes: are the above inclusions strict?

In this paper we give a sufficient and necessary condition for an analytic function with Hadamard gaps to belong to $B^{p}$ or $B_{0}^{p}$. In view of the result it is easy to conclude that the above inclusions (7) and (8) are strict. Hence we get a negative answer to the question in general.

\section{Main Result}

Our main result is the following theorem.

THEOREM 1. Let $0<p<\infty$. If $f(z)=\sum_{k=1}^{\infty} a_{k} z^{n_{k}}$ is analytic on $D$ and has Hadamard gaps, that is, if

$$
\frac{n_{k+1}}{n_{k}} \geqslant \lambda>1, \quad(k=1,2, \ldots)
$$

then the following statements are equivalent:

$$
\text { (I) } f \in B^{p} ; \quad \text { (II) } f \in B_{0}^{p} ; \quad \text { (III) } \sum_{k=1}^{\infty}\left|a_{k}\right|^{p}<\infty .
$$

By Theorem 1 we can give the answer to the question in the introduction. Let $0<p<2$. Then $f(z)=\sum_{n=1}^{\infty}\left(z^{2^{n}}\right) /\left(n^{1 / p}\right) \in V M O A$, but $f \notin B^{p}$. Let $2<q<\infty$. Then $g(z)=\sum_{n=1}^{\infty}\left(z^{2^{n}}\right) /\left(n^{1 / 2}\right) \in B_{0}^{q}$, but $g \notin B M O A$. Hence the inclusions (7) and (8) are strict. Furthermore we know that the following inclusions, for $0<p<q<\infty$,

$$
B^{p} \subset B^{q} ; \quad B_{0}^{p} \subset B_{0}^{q},
$$

are strict.

In order to prove Theorem 1, we need the following two lemmas. 
LеммA 1. Let $0<p<\infty$. If $\left\{n_{k}\right\}$ is an increasing sequence of positive integers satisfying $n_{k+1} / n_{k} \geqslant \lambda>1$ for all $k$, then there is a constant $A$ depending only on $p$ and $\lambda$ such that

$$
A^{-1}\left(\sum_{k=1}^{\infty}\left|a_{k}\right|^{2}\right)^{1 / 2} \leqslant\left(\frac{1}{2 \pi} \int_{0}^{2 \pi}\left|\sum_{k=1}^{\infty} a_{k} e^{i n_{k} \theta}\right|^{p} d \theta\right)^{1 / p} \leqslant A\left(\sum_{k=1}^{\infty}\left|a_{k}\right|^{2}\right)^{1 / 2}
$$

for any number $a_{k}(k=1,2, \ldots)$.

The above lemma was due to Zygmund [5].

LEMMA 2. Let $\alpha>0, p>0, n \geqslant 0, a_{n} \geqslant 0, I_{n}=\left\{k: 2^{n} \leqslant k<2^{n+1}, k \in N\right\}$, $t_{n}=\sum_{k \in I_{n}} a_{k}$ and $f(x)=\sum_{n=1}^{\infty} a_{n} x^{n}$. Then there is a constant $K$ depending only on $p$ and $\alpha$ such that

$$
\frac{1}{K} \sum_{n=0}^{\infty} 2^{-n \alpha} t_{n}^{p} \leqslant \int_{0}^{1}(1-x)^{\alpha-1} f(x)^{p} d x \leqslant K \sum_{n=0}^{\infty} 2^{-n \alpha} t_{n}^{p} .
$$

The proof of Lemma 2 can be found in [3]. By simple computation we see that the above lemma is still valid for $f(x)=\sum_{n=1}^{\infty} a_{n} x^{n-1}, a_{n} \geqslant 0$. Let $K$ still denote the constant in Lemma 2 for $f(x)=\sum_{n=1}^{\infty} a_{n} x^{n-1}$.

For our purpose we will use the following inequalities, which follow immediately from Hölder's inequality. Let $a_{n} \geqslant 0$ and let $N$ be a positive integer. Then for $0<p \leqslant 1$,

$$
\frac{1}{N^{1-p}}\left(\sum_{n=1}^{N} a_{n}^{p}\right) \leqslant\left(\sum_{n=1}^{N} a_{n}\right)^{p} \leqslant\left(\sum_{n=1}^{N} a_{n}^{p}\right)
$$

for $1 \leqslant p<\infty$,

$$
\left(\sum_{n=1}^{N} a_{n}^{p}\right) \leqslant\left(\sum_{n=1}^{N} a_{n}\right)^{p} \leqslant N^{p-1}\left(\sum_{n=1}^{N} a_{n}^{p}\right) .
$$

Before proving Theorem 1 we first prove the following result, which is useful for the proof of Theorem 1 and is of independent interest. We state it as a theorem.

THEOREM 2. Let $0<p<\infty, I_{n}=\left\{k: 2^{n} \leqslant k<2^{n+1}, k \in N\right\}$ and let $f(z)=\sum_{n=0}^{\infty} a_{n} z^{n}$ be analytic on $D$. If

$$
\sum_{n=0}^{\infty}\left(\sum_{k \in I_{n}}\left|a_{k}\right|\right)^{p}<\infty
$$


then $f \in B_{0}^{p}$.

Proof: By the following identity:

$$
1-\left|\varphi_{\lambda}(z)\right|^{2}=\frac{\left(1-|\lambda|^{2}\right)\left(1-|z|^{2}\right)}{|1-\bar{\lambda} z|^{2}}, \quad(\lambda, z \in D)
$$

we have

$$
\begin{aligned}
\int_{D}\left|f^{\prime}(z)\right|^{p} & \left(1-|z|^{2}\right)^{p-2}\left(1-\left|\varphi_{\lambda}(z)\right|^{2}\right) d A(z) \\
& \leqslant \int_{D}\left(\sum_{n=1}^{\infty} n\left|a_{n}\right||z|^{n-1}\right)^{p} \frac{\left(1-|z|^{2}\right)^{p-1}\left(1-|\lambda|^{2}\right)}{|1-\bar{\lambda} z|^{2}} d A(z) \\
& =\int_{0}^{1}\left(\sum_{n=1}^{\infty} n\left|a_{n}\right| r^{n-1}\right)^{p}\left(1-r^{2}\right)^{p-1}\left(1-|\lambda|^{2}\right)\left(\int_{0}^{2 \pi} \frac{d \theta}{\left|1-\bar{\lambda} r e^{i \theta}\right|^{2}}\right) r d r \\
& =2 \pi \int_{0}^{1}\left(\sum_{n=1}^{\infty} n\left|a_{n}\right| r^{n-1}\right)^{p}\left(1-r^{2}\right)^{p-1} \frac{1-|\lambda|^{2}}{1-|\lambda|^{2} r^{2}} r d r \\
& \leqslant 2^{p} \pi \int_{0}^{1}\left(\sum_{n=1}^{\infty} n\left|a_{n}\right| r^{n-1}\right)^{p}(1-r)^{p-1} d r \\
& \leqslant 2^{p} \pi K \sum_{n=0}^{\infty} 2^{-n p} t_{n}^{p},
\end{aligned}
$$

because of Lemma 2, where

$$
t_{n}=\sum_{k \in I_{n}} k\left|a_{k}\right|<2^{n+1} \sum_{k \in I_{n}}\left|a_{k}\right| .
$$

Then we get

$$
\begin{aligned}
\|f\|_{B^{p}}^{p} & =\sup _{\lambda \in D} \int_{D}\left|f^{\prime}(z)\right|^{p}\left(1-|z|^{2}\right)^{p-1} \frac{1-|\lambda|^{2}}{|1-\bar{\lambda} z|^{2}} d A(z) \\
& \leqslant 4^{p} \pi K \sum_{n=0}^{\infty}\left(\sum_{k \in I_{n}}\left|a_{k}\right|\right)^{p}<\infty,
\end{aligned}
$$

that is, $f \in B^{p}$. To prove that $f \in B_{0}^{p} \subset B^{p}$, we note that the integral $\int_{0}^{1}\left(\sum_{n=1}^{\infty} n\left|a_{n}\right| r^{n-1}\right)^{p}\left(1-r^{2}\right)^{p-1} d r$ is convergent, for $\sum_{n=0}^{\infty}\left(\sum_{k \in I_{n}}\left|a_{k}\right|\right)^{p}<\infty$. Hence for any $\varepsilon>0$, there is a $\delta \in(0,1)$ such that

$$
\int_{\delta}^{1}\left(\sum_{n=1}^{\infty} n\left|a_{n}\right| r^{n-1}\right)^{p}\left(1-r^{2}\right)^{p} d r<\varepsilon
$$


Then

$$
\begin{aligned}
\int_{D}\left|f^{\prime}(z)\right|^{p} & \left(1-|z|^{2}\right)^{p-1} \frac{1-|\lambda|^{2}}{|1-\bar{\lambda} z|^{2}} d A(z) \\
& \leqslant 2 \pi \int_{0}^{1}\left(\sum_{n=1}^{\infty} n\left|a_{n}\right| r^{n-1}\right)^{p}\left(1-r^{2}\right)^{p-1} \frac{1-|\lambda|^{2}}{1-|\lambda|^{2} r^{2}} d r \\
& <2 \pi \int_{0}^{\delta}\left(\sum_{n=1}^{\infty} n\left|a_{n}\right| r^{n-1}\right)^{p}\left(1-r^{2}\right)^{p-1} \frac{1-|\lambda|^{2}}{1-|\lambda|^{2} r^{2}} d r+2 \pi \varepsilon \\
& <2 \pi \frac{1-|\lambda|^{2}}{1-\delta^{2}} \int_{0}^{1}\left(\sum_{n=1}^{\infty} n\left|a_{n}\right| r^{n-1}\right)^{p}\left(1-r^{2}\right)^{p-1} d r+2 \pi \varepsilon
\end{aligned}
$$

If $|\lambda|$ is chosen appropriately so $1-|\lambda|$ may be sufficiently small, then the above quantity can be less than $4 \pi \varepsilon$. Hence

$$
\lim _{|\lambda| \rightarrow 1-0} \int_{D}\left|f^{\prime}(z)\right|^{p}\left(1-|z|^{2}\right)^{p-2}\left(1-\left|\varphi_{\lambda}(z)\right|^{2}\right) d A(z)=0 .
$$

According to definition (6), it follows that $f \in B_{0}^{p}$. This completes the proof.

Proof of Theorem 1: It is clear that (II) implies (I). We first prove that (III) follows from (I). Applying Lemma 1 and Lemma 2 we get

$$
\begin{aligned}
\|f\|_{B^{p}}^{p} & \geqslant \int_{D}\left|f^{\prime}(z)\right|^{p}\left(1-|z|^{2}\right)^{p-1} d A(z) \\
& =\int_{D}\left|\sum_{k=1}^{\infty} n_{k} a_{k} z^{n_{k}-1}\right|^{p}\left(1-|z|^{2}\right)^{p-1} d A(z) \\
& \geqslant \frac{2 \pi}{A^{p}} \int_{0}^{1}\left(1-r^{2}\right)^{p-1}\left(\sum n_{k}^{2}\left|a_{k}\right|^{2} r^{2\left(n_{k}-1\right)}\right)^{p / 2} r d r \\
& \geqslant \frac{\pi}{A^{p}} \int_{0}^{1}(1-x)^{p-1}\left(\sum_{k=1}^{\infty} n_{k}^{2}\left|a_{k}\right|^{2} x^{n_{k}}\right)^{p / 2} d x \\
& \geqslant \frac{\pi}{K A^{p}} \sum_{k=0}^{\infty} 2^{-k p} t_{k}^{p / 2}
\end{aligned}
$$

where

$$
t_{k}=\sum_{n_{j} \in I_{k}} n_{j}^{2}\left|a_{j}\right|^{2} .
$$

Because $n_{k+1} / n_{k} \geqslant \lambda>1$ for all $k$, the number of Taylor coefficients $a_{j}$ is at most $\left[\log _{\lambda} 2\right]+1$ when $n_{j} \in I_{k}$, for $k=1,2, \ldots$ Then

$$
t_{k}^{p / 2} \geqslant 2^{k p} C_{p} \sum_{n_{j} \in I_{k}}\left|a_{j}\right|^{p}
$$


where $C_{p}=1$ for $p / 2 \geqslant 1$ and $C_{p}=1 /\left(\left[\log _{\lambda} 2\right]+1\right)^{1-p / 2}$ for $p / 2<1$, by (9) and (10). Combining the above inequalities yields that (III) holds.

By Theorem 2 it is easy to prove that (II) follows from (III). Assuming that $\sum_{k=1}^{\infty}\left|a_{k}\right|^{p}<\infty$ and $n_{k+1} / n_{k} \geqslant \lambda>1$ for all $k$, we have

$$
\sum_{n=0}^{\infty}\left(\sum_{n_{k} \in I_{n}}\left|a_{k}\right|\right)^{p} \leqslant\left(\left[\log _{\lambda} 2\right]+1\right)^{p} \sum_{k=1}^{\infty}\left|a_{k}\right|^{p}<\infty,
$$

by (9) and (10). Thus $f \in B_{0}^{p}$, and the proof is complete.

Theorem 1 should be compared with the following result (see [1]):

THEOREM A. Let $f(z)=\sum_{k=1}^{\infty} a_{k} z^{n_{k}}$ be analytic on $D$. If $f$ has Hadamard gaps, then $f \in \mathcal{B}$ if and only if $a_{k}=0(1)(k \rightarrow \infty)$; and $f \in \mathcal{B}_{0}$ if and only if $a_{k} \rightarrow 0$ $(k \rightarrow \infty)$.

Setting $p=2$ in Theorem 2, we have

COROLLARY. Let $f(z)=\sum_{n=0}^{\infty} a_{n} z^{n}$ be analytic on $D$. If

$$
\sum_{n=0}^{\infty}\left(\sum_{j \in I_{n}}\left|a_{j}\right|\right)^{2}<\infty
$$

then $f \in V M O A$.

REMARK. By (10) we have

thus

$$
\left(\sum_{j \in I_{n}}\left|a_{j}\right|\right)^{2} \leqslant 2^{n} \sum_{j \in I_{n}}\left|a_{j}\right|^{2} \leqslant \sum_{j \in I_{n}} j\left|a_{j}\right|^{2},
$$

$$
\sum_{n=0}^{\infty}\left(\sum_{j \in I_{n}}\left|a_{j}\right|\right)^{2} \leqslant \sum_{n=1}^{\infty} n\left|a_{n}\right|^{2}=\frac{1}{\pi} \int_{D}\left|f^{\prime}(z)\right|^{2} d A(z) .
$$

Hence the condition in the Corollary is weaker than

$$
\int_{D}\left|f^{\prime}(z)\right|^{2} d A(z)<\infty
$$

\section{REFERENCES}

[1] J.M. Anderson, J. Clunie and Ch. Pommerenke, 'On Bloch functions and normal functions', J. Reine Angew. Math. 270 (1974), 12-37. 
[2] J.B. Garnett, Bounded analytic functions (Academic Press, New York, 1981).

[3] M. Mateljević and M. Pavlović, ' $L^{P}$-behavior of power series with positive coefficients and Hardy spaces', Proc. Amer. Math. Soc. 87 (1983), 309-316.

[4] K. Stroethoff, 'Besov-type characterisations for the Bloch space', Bull. Austral. Math. Soc. 39 (1989), 405-420.

[5] A. Zygmund, Trigonometric series (Cambridge Univ. Press, London, 1959).

\footnotetext{
Department of Mathematics

Hangzhou University

Hangzhou, Zhejiang

Peoples Republic of China
} 